\title{
Sepsis and the orexin system
}

\author{
Kazuyoshi Hirota ${ }^{1}$
}

Received: 1 June 2016 / Accepted: 24 August 2016 / Published online: 31 August 2016

(C) Japanese Society of Anesthesiologists 2016

\section{Introduction}

The definition of sepsis was revised in Feb 2016 [1]. However, sepsis remains one of the leading causes of death worldwide. Twenty to 30 million patients suffer from sepsis and approximately one-third of these die every year. Early recognition and standardized emergency treatment such as appropriate antibiotic therapy are essential [2]. However, early recognition is not always easy as typical signs and symptoms are not always obvious in all critically ill patients. There has been a concerted effort from both clinical and basic researchers to address this issue of recognition with several studies attempting to establish early diagnostic criteria [3-5].

Several reports suggest that neuronal bioactive substances such as nociceptin [4] and endocanabinoid [6] may contribute to the pathophysiology of sepsis. Several reports [7-9] suggest that orexin (OX) may also be involved. Degeneration of orexinergic (OXergic) neurons occurs during sepsis and the decline of OXergic activity may partly characterize the symptoms of sepsis. Here, we discuss the relationship between the OXergic system and sepsis.

\section{Orexins and orexin receptors}

Orexin-A [OXA: molecular weight $(\mathrm{MW}$, human $)=3561.10$ ] and $-\mathrm{B}$ [OXB: MW (human) $=2899.34]$ are endogenous neuropeptide agonists for orexin-1 (OX1) and orexin-2 (OX2)

Kazuyoshi Hirota

hirotak@hirosaki-u.ac.jp

1 Department of Anesthesiology, Hirosaki University Graduate School of Medicine, Hirosaki 036-8562, Japan receptors [10], and are synthesized mainly by neurons located in the posterolateral hypothalamus. OX1 receptors are widely distributed in the central nervous system (CNS), mostly in the ventromedial hypothalamus, and in peripheral tissues such as brown adipose tissue. OX2 receptors are also widely distributed in the CNS, mainly in the paraventricular nucleus, and in peripheral tissues such as the adrenal medulla [11]. The OXergic system plays an important role in several physiological functions such as feeding, sleep-wake cycle, locomotion, thermoregulation, autonomic function including cardiovascular function, and analgesia. OXergic system is particularly known to be involved in the control of the sympathetic nervous system [12]. In addition, the OXergic system contributes to the cardiovascular defense system which is attenuated in OX knockout mice and OX neuron-ablated mice [13].

\section{Pathophysiology of sepsis and the OXergic system}

\section{Sleep disorder}

Patients with sepsis in the intensive care unit (ICU) often show complete absence of the normal circadian rhythm pattern. Although rapid eye movement (REM) sleep typically occupies $20-25 \%$ of nocturnal sleep in healthy subjects, patients with sepsis often show an absence or a decrease in REM sleep. It has been suggested that some specific mediators such as endotoxin may disrupt REM sleep [14]. Baracchi et al. [15] reported that septic rats showed suppression of REM sleep and $\delta$-power during non-REM sleep and fragmented sleep during dark periods. Lipopolysaccharide (LPS) infusion significantly decreases OXergic neurons [7-9]. LPS infusion at $0.22 \mu \mathrm{g} / \mathrm{h}$ significantly reduced not only OX by $29.7 \%$ but also REM sleep [7]. The sleepwake cycle returns to normal after recovery from sepsis. 
Palomba et al. [9] found that OX expression declined after LPS infusion and completely recovered to the pre-LPS level 30 days after the last LPS injection.

\section{Sepsis-associated delirium}

Delirium is evident in $50 \%$ of patients with sepsis in the ICU. Clinical features of sepsis-associated delirium (SAD) are altered level of consciousness, decrease in attention, changes in cognition and perceptual disturbances. SAD may be due to neuroinflammation, abnormal cerebral perfusion and neurotransmitter imbalance [16]. Indeed, some septic patients reveal EEG changes indicating mild to moderate encephalopathy [7]. SAD is generally reversible as the symptoms usually disappear after the successful treatment of sepsis [16].

OXergic neuronal activity has been implicated in the modulation of learning and memory as OXergic neurons project to the hippocampus, which is the main seat of learning and memory. As ischemic stroke results in inflammation in the brain that directly affects the repair of neural damage, stroke patients often exhibit cognitive impairment within 3 months of a stroke with a reduction in serum and cerebrospinal fluid OX concentrations [17]. In addition, nasal administration of OXA improved cognitive performance in sleep-deprived nonhuman primates [18] and social memory in adult OX/ataxin-3-transgenic mice [19]. These evidences suggest that the OXergic system may play an important role in cognitive functions. Therefore, a reduction in OXergic neurons during sepsis may also contribute to SAD.

A recent article [20] suggested that hyperprolactinemia may also be associated with SAD. As prolactin can increase cytokine release from astrocytes and aggravate brain endothelial dysfunction, excessive prolactin release may exert some harmful effects on the brain and cause cognitive dysfunction [20]. It is also reported that the hypothalamic expression of OX is significantly decreased with high plasma levels of prolactin such as in pregnancy and lactation [21]. In addition, OX can regulate prolactin secretion [22]. Therefore, an increase in prolactin secretion during sepsis might participate in neuroinflammation-caused SAD with a decline of OXergic neurons.

\section{Nutrition}

Appetite loss and gastrointestinal (GI) dysmotility are often accompanied by chronic inflammatory conditions including sepsis [23]. Sepsis is one of the risk factors for gastroparesis and ileus in the ICU [23]. Hyperglycemia is also present in patients with sepsis as insulin resistance is associated with an increase in plasma LPS concentration, which is derived from the membrane of Gram-negative bacteria in the GI tract [24].
OXA, OXB and their receptors are distributed not only in the CNS but also in peripheral tissues including the intestine and pancreas, i.e., organs that are important for glucose homeostasis [25]. It has been reported that depression of the OXergic system produces appetite loss and disturbance of GI function [26]. OXergic activity is stimulated by low glucose $(2.8 \mathrm{mM})$ and inhibited by high glucose $(16.7 \mathrm{mM})$. As OX could inhibit the active absorption of luminal glucose in the intestine, OX directly modulates glucose disposal in the GI tract, although the peripheral role of OX in glucose homeostasis has not fully been elucidated [25]. On the other hand, the expression of OX in the hypothalamus is reduced by hyperglycemia due to insulin insensitivity [25]. Thus, not only inflammation but also hyperglycemia may contribute to the depression of OXergic activity in sepsis.

\section{Hemodynamics}

Cardiovascular dysfunction profoundly affects the outcome of sepsis. Moreover, sepsis also induces autonomic dysregulation. Sepsis-related cardiac depression may be related to lower responsiveness to endogenous catecholamines because of disrupted signal transduction with a reduction in adrenoceptor density and an increase in inhibitory G-protein in the myocardium despite high plasma catecholamines. In addition, septic shock is associated with neuronal and glial apoptosis within cardiac autonomic centers [27].

Central administration of OXs can increase blood pressure, heart rate and sympathetic activity as OXergic neurons are located in the hypothalamus with distribution of their terminals in the central autonomic regions [28]. We also found that OXergic neurons could regulate the sympathetic nervous system as OX/ataxin-3 transgenic (OX neuron-ablated) rats showed decreased sympathetic nerve tone. OX/ataxin-3 rats also showed lower systolic blood pressure than wild-type rats [12]. Thus, degeneration of OXergic neurons might be partially involved in the mechanism of cardiovascular dysfunction in sepsis.

\section{Anesthetic requirement}

Several animal studies indicate that sepsis could reduce the requirement of both volatile (sevoflurane, desflurane and isoflurane) [29] and intravenous anesthetics (propofol) [30]. In these studies, although it was well-known that anesthetic requirement could be altered by physiological conditions such as body temperature, age, acid-base balance, electrolyte balance, and blood pressure, the physiologic conditions in the sepsis group were adjusted to those in the control group. Therefore, the reduction in anesthetic requirement may be due to other factors such as CNS dysfunction. 
As mentioned above, sepsis depresses OXergic neurons. In addition, we previously reported that intracerebroventricular (i.c.v.) administration of OXs reduced intravenous anesthesia time while OX1 receptor antagonist prolonged it [31, 32]. Similarly, Kelts et al. [33] reported that genetic ablation of OXergic neurons (OX/ataxin-3 mice) and selective OX1 receptor antagonists delayed emergence from volatile anesthesia such as sevoflurane and isoflurane. Based on these observations, sepsis-induced OXergic depression may reduce anesthesia requirement.

\section{Therapeutic potential of OX receptor agonists in sepsis}

Similar to sepsis, inflammatory brain diseases such as Alzheimer's disease, Parkinson's disease, traumatic brain injury and stroke reveal OX deficiency. In inflammatory brain disease models, glucagon-like peptide-1 (GLP-1) mimetics have been reported to reverse memory impairment, synaptic and neuronal loss, and behavioral dysfunction [34]. As GLP-1 mimetics have been reported to activate OXergic neurons in ex vivo hypothalamic slices, GLP-1 mimetics could be functional OX receptor agonists. In a post-chemotherapy model, rodents revealed fatigue which is defined as a significant decline in voluntary locomotor activity. In addition, cytotoxic chemotherapy produced hypothalamic inflammation and suppressed hypothalamic OXergic activity, and this fatigue was reversed by i.c.v. OXA [34]. Thus, the pathophysiologic symptoms of sepsis might be improved by an increase in OXergic activity. Indeed, i.c.v. OXA improved arousal, temperature, and heart and respiratory rates when OXergic activity was significantly reduced to one-sixth of baseline in a septic state induced by cecal ligation and puncture [34]. However, as there has only been this one report to suggest that i.c.v. OXA could improve the pathogenesis of sepsis, more animal experiments will be required for confirmation. In addition, clinical investigations will also be required.

\section{Conclusion}

Various neuronal and immune systems participate in the complicated clinical features of sepsis. However, reported data indicate that the OXergic system must be involved in the pathophysiology of sepsis. It is necessary to know what are the precise sites and how they might be clinically effectively targeted. What is clear is that beyond supportive therapy and antibiotic use there are no wonder drugs available for use in the ICU: could targeting the OXergic system represent a new therapeutic approach?

\section{References}

1. Shankar-Hari M, Phillips GS, Levy ML, Seymour CW, Liu VX, Deutschman CS, Angus DC, Rubenfeld GD, Singer M, Sepsis Definitions Task Force. Developing a new definition and assessing new clinical criteria for septic shock: for the third international consensus definitions for sepsis and septic shock (sepsis-3). JAMA. 2016;315:775-87.

2. Reinhart K, Daniels R, Kissoon N, O'Brien J, Machado FR, Jimenez E, GSA Executive Board and WSD Executive Board. The burden of sepsis-a call to action in support of world sepsis day 2013. J Crit Care. 2013;28:526-8.

3. Sankar V, Webster NR. Clinical application of sepsis biomarkers. J Anesth. 2013;27:269-83.

4. Thomas R, Stover C, Lambert DG, Thompson JP. Nociceptin system as a target in sepsis? J Anesth. 2014;28:759-67.

5. Saito J, Hashiba E, Kushikata T, Mikami A, Hirota K. Changes in presepsin concentrations in surgical patients with end-stage kidney disease undergoing living kidney transplantation: a pilot study. J Anesth. 2016;30:174-7.

6. Sardinha J, Kelly ME, Zhou J, Lehmann C. Experimental cannabinoid 2 receptor-mediated immune modulation in sepsis. Mediators Inflamm. 2016. doi:10.1155/2014/978678.

7. Gerashchenko D, Shiromani PJ. Effects of inflammation produced by chronic lipopolysaccharide administration on the survival of hypocretin neurons and sleep. Brain Res. 2004;1019:162-9.

8. Perekrest SV, Abramova TV, Novikova NS, Loskutov YV, Rogers VJ, Korneva EA. Changes in immunoreactivity of orexin-Apositive neurons after intravenous lipopolysaccharide injection. Med Sci Monit. 2008;14:BR127-33.

9. Palomba M, Seke Etet PF, Veronesi C. Effect of inflammatory challenge on hypothalamic neurons expressing orexinergic and melanin-concentrating hormone. Neurosci Lett. 2014;570:47-52.

10. Hirota K, Kushikata T, Yoshida H, Kudo M, Kudo T. Role of the orexinergic system in acute haemorrhage in the rat. Neurosci Lett. 2008;432:162-6.

11. Mikulášková B, Maletínská L, Zicha J, Kuneš J. The role of food intake regulating peptides in cardiovascular regulation. Mol Cell Endocrinol. 2016;436:78-92.

12. Murakami M, Ohba T, Kushikata T, Niwa H, Kurose A, Imaizumi T, Watanabe H, Yanagisawa T, Nakaji S, Ono K, Hirota K. Involvement of the orexin system in sympathetic nerve regulation. Biochem Biophys Res Commun. 2015;460:1076-81.

13. Zhang W, Shimoyama M, Fukuda Y, Kuwaki T. Multiple components of the defense response depend on orexin: evidence from orexin knockout mice and orexin neuron-ablated mice. Auton Neurosci. 2006;126-7:139-45.

14. Freedman NS, Gazendam J, Levan L, Pack AI, Schwab RJ. Abnormal sleep/wake cycles and the effect of environmental noise on sleep disruption in the intensive care unit. Am J Respir Crit Care Med. 2001;163:451-7.

15. Baracchi F, Ingiosi AM, Raymond RM Jr, Opp MR. Sepsisinduced alterations in sleep of rats. Am J Physiol Regul Integr Comp Physiol. 2011;301:R1467-78.

16. Piva S, McCreadie VA, Latronico N. Neuroinflammation in sepsis: sepsis associated delirium. Cardiovasc Hematol Disord: Drug Targets. 2015;15:10-8.

17. Song J, Kim E, Kim CH, Song HT, Lee JE. The role of orexin in post-stroke inflammation, cognitive decline, and depression. Mol Brain. 2015;8:16.

18. Deadwyler SA, Porrino L, Siegel JM, Hampson RE. Systemic and nasal delivery of orexin-A (Hypocretin-1) reduces the effects of sleep deprivation on cognitive performance in nonhuman primates. J Neurosci. 2007;27:14239-47. 
19. Yang L, Zou B, Xiong X, Pascual C, Xie J, Malik A, Xie J, Sakurai T, Xie XS. Hypocretin/orexin neurons contribute to hippocampus-dependent social memory and synaptic plasticity in mice. J Neurosci. 2013;33:5275-84.

20. Nguyen DN, Huyghens L, Schiettecatte J, Smitz J, Vincent JL. High prolactin levels are associated with more delirium in septic patients. J Crit Care. 2016;33:56-61.

21. García MC, López M, Gualillo O, Seoane LM, Diéguez C, Señarís RM. Hypothalamic levels of NPY, MCH, and prepro-orexin mRNA during pregnancy and lactation in the rat: role of prolactin. FASEB J. 2003;17:1392-400.

22. Zieba DA, Misztal T, Romanowicz K, Wszola M, Wierzchos E, Nowakowski M. The role of orexin A in the control of prolactin and growth hormone secretions in sheep-in vitro study. J Physiol Pharmacol. 2008;59(Suppl 9):91-100.

23. Aderinto-Adike AO, Quigley EM. Gastrointestinal motility problems in critical care: a clinical perspective. J Dig Dis. 2014;15:335-44.

24. Boroni Moreira AP, de Cássia Gonçalves Alfenas R. The influence of endotoxemia on the molecular mechanisms of insulin resistance. Nutr Hosp. 2012;27:382-90.

25. Tsuneki H, Wada T, Sasaoka T. Role of orexin in the regulation of glucose homeostasis. Acta Physiol. 2010;198:335-48.

26. Okumura T, Nozu T. Role of brain orexin in the pathophysiology of functional gastrointestinal disorders. J Gastroenterol Hepatol. 2011;26(Suppl 3):61-6.
27. Antonucci E, Fiaccadori E, Donadello K, Taccone FS, Franchi F, Scolletta S. Myocardial depression in sepsis: from pathogenesis to clinical manifestations and treatment. J Crit Care. 2014;29:500-11.

28. Carrive P. Orexin, orexin receptor antagonists and central cardiovascular control. Front Neurosci. 2013;7:257.

29. Allaouchiche B, Duflo F, Tournadre JP, Debon R, Chassard D. Influence of sepsis on sevoflurane minimum alveolar concentration in a porcine model. Br J Anaesth. 2001;86:832-6.

30. Bollen PJ, Nielsen BJ, Toft P. Influence of endotoxin-induced sepsis on the requirements of propofol-fentanyl infusion rate in pigs. Basic Clin Pharmacol Toxicol. 2007;101:192-6.

31. Kushikata T, Hirota K, Yoshida H, Kudo M, Lambert DG, Smart D, Jerman JC, Matsuki A. Orexinergic neurons and barbiturate anesthesia. Neuroscience. 2003;121:855-63.

32. Tose R, Kushikata T, Yoshida H, Kudo M, Furukawa K, Ueno S, Hirota K. Orexin A decreases ketamine-induced anesthesia time in the rat: the relevance to brain noradrenergic neuronal activity. Anesth Analg. 2009;1082:491-5.

33. Kelz MB, Sun Y, Chen J, Cheng Meng Q, Moore JT, Veasey SC, Dixon S, Thornton M, Funato H, Yanagisawa M. An essential role for orexins in emergence from general anesthesia. Proc Natl Acad Sci USA. 2008;105:1309-14.

34. Clark IA, Vissel B. Inflammation-sleep interface in brain disease: TNF, insulin, orexin. J Neuroinflammation. 2014;11:51. 